\title{
Advances in Preclinical SPECT Instrumentation
}

\author{
Todd E. Peterson ${ }^{1-3}$ and Sepideh Shokouhi ${ }^{1,2}$ \\ ${ }^{1}$ Institute of Imaging Science, Vanderbilt University, Nashville, Tennessee; ${ }^{2}$ Department of Radiology and Radiological Sciences, \\ Vanderbilt University, Nashville, Tennessee; and ${ }^{3}$ Department of Physics and Astronomy, Vanderbilt University, Nashville, Tennessee
}

Preclinical SPECT of rodents is both in demand and very demanding. The need for high spatial resolution in combination with good sensitivity has given rise to considerable innovation in the areas of detectors, collimation, acquisition geometry, and image reconstruction. Some of the developments described herein are beginning to carry over into clinical imaging as well.

Key Words: SPECT; preclinical imaging; semiconductor detectors; multi-pinhole collimation; synthetic collimator

J Nucl Med 2012; 53:841-844

DOI: 10.2967/jnumed.111.099853

Preclinical imaging of small animals plays an important role not only in the development of new imaging agents but also in biologic studies of both disease and nondisease states as well as in drug development. Although in vivo preclinical studies pave the way for new clinical applications of molecular imaging, they also provide an important testing ground for new instrumentation and image acquisition strategies that themselves have the potential to transform clinical molecular imaging. The focus of this article will be on innovation in preclinical SPECT, although other modalities have seen similar developments. To image organs or structures within a mouse with a quality similar to that in a human requires improvements in both spatial resolution and sensitivity over clinical SPECT. Attempting to meet the sensitivity-resolution demands of preclinical SPECT has led to innovation in collimation, detectors, calibration, and image reconstruction. In this brief article, we will survey these developments through the lens of our own research efforts.

\section{COLLIMATION}

Multipinhole collimation has become the standard approach in most preclinical SPECT systems because of the ability to obtain good spatial resolution through pinhole magnification while enhancing sensitivity though the incorporation of multiple pinholes. In the standard formulation, the planar spatial resolution of a pinhole is

$$
R_{\text {total }}=\sqrt{d^{2}\left(1+\frac{1}{M}\right)^{2}+R_{d e t}^{2} / M^{2}}
$$

Received Mar. 26, 2012; revision accepted May 1, 2012.

For correspondence or reprints contact: Todd E. Peterson, Vanderbilt University Institute of Imaging Sciences (VUIIS), Vanderbilt University Medical School, 116121 st Ave. S., AA 1105 MCN, Nashville, TN 37232-2310.

E-mail: todd.e.peterson@vanderbilt.edu

Published online May 14, 2012.

COPYRIGHT $\odot 2012$ by the Society of Nuclear Medicine, Inc. where $d$ is the pinhole diameter, $M$ is the magnification, and $R_{d e t}$ is the intrinsic detector resolution. The sensitivity to a point source (fraction of emitted photons passing through the pinhole) is given by

$$
S=\frac{d^{2}}{16 h^{2}} \cos ^{3} \theta
$$

where $h$ is the perpendicular distance from the pinhole, and $\theta$ is the angle between the point-to-pinhole projection line and the normal to the detector. The sensitivity-resolution tradeoff for pinhole collimation is immediately apparent. Although the spatial resolution scales approximately linearly with pinhole diameter, the sensitivity is proportional to the square of the pinhole diameter; shrinking the pinhole diameter to improve the spatial resolution comes with a steep penalty in sensitivity.

These simplified equations ignore several effects, such as details of pinhole geometry and photon penetration at pinhole edges. Depending on the collimator design, acquisition geometry, and photon energy, these effects can become sufficiently large that their inclusion is necessary to accurately model pinhole projections. The amount of pinhole penetration depends on the photon energy and collimator material and represents a limiting factor in spatial resolution. ${ }^{125}$ I-labeled radiotracers are sometimes used in preclinical SPECT, and their low photon energy limits pinhole penetration, reducing the effective pinhole diameter and enabling better resolution relative to higher-energy radionuclides. The geometric sensitivity in Equation 2 does not explicitly include the pinhole opening angle, shape, or thickness. Deviation from this expression has been shown to occur with the use of small pinhole opening angles, which reduce penetration, and short object-to-collimator distances, which improve sensitivity and resolution (1), and consideration of such effects can be important in the design of new systems.

Increasing the number of pinholes in a collimator can either improve the sensitivity and angular sampling for a fixed field of view or enlarge the field of view, depending on the choice of pinhole positions and focal points. The primary limitation in multipinhole collimator design is the overlap of the projections from different pinholes. This so-called multiplexing creates ambiguity as to which pinhole a detected photon may have passed through, reducing the sensitivity advantage of the added pinholes and possibly leading to artifacts in the reconstructed images. The amount of multiplexing and its effect on image reconstruction has been the subject of some studies on multipinhole collimator design $(2,3)$. Although some preclinical scan- 
ners, such as the U-SPECT system from MILabs (4), use multipinhole collimation with conventional $\gamma$-cameras and no multiplexing to avoid any potential problems, the NanoSPECT from Bioscan delivers high-quality images despite allowing some multiplexing of projections (5). Rentmeester et al. (6) investigated the optimal multipinhole SPECT configuration when no multiplexing is allowed, but the larger parameter space created when multiplexing is allowed makes for a daunting optimization problem. In simulation studies in which only the number of pinholes in a fixed geometric configuration was varied, Cao et al. found that a camera with a 9-pinhole collimator gave the best performance for their quantitative mouse brain-imaging task (7). Importantly, changes in the geometric configuration of the pinholes would likely change the outcome, because of differences in angular sampling and multiplexing, and Mok et al. demonstrated that the performance of a multiplexed multipinhole system also depends on the object activity distribution (3).

Our approach to increasing sensitivity through the use of multipinhole collimation has been to allow multiplexing but to acquire data in such a way that the data contain information about the multiplexing. This approach is known as synthetic collimation and involves the acquisition of pinhole projection data at multiple magnifications (Fig. 1) $(8,9)$. The basic concept behind the synthetic collimator approach is to combine lowerresolution data containing little (or no) multiplexing with higher-resolution data that are more highly multiplexed. The lower-magnification data then contain information about the nature of the multiplexing in the higher-magnification data such that, when combined in an appropriate image reconstruction algorithm, SPECT images largely free of artifacts can be generated. How best to choose the pinhole overlap and magnifications is the subject of ongoing investigations (9).

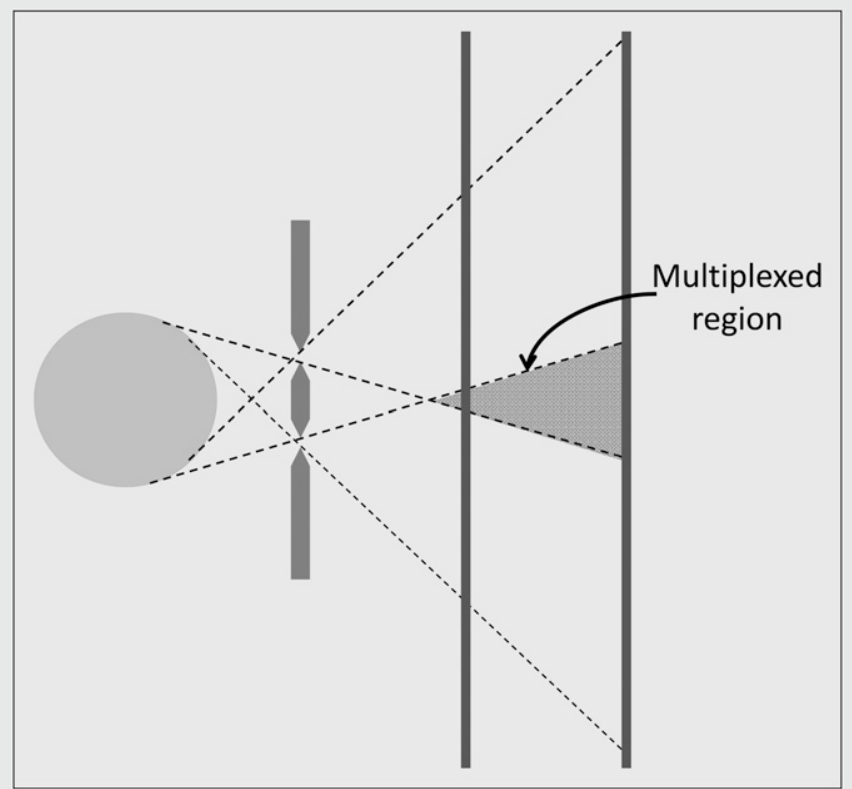

FIGURE 1. In synthetic collimator imaging, multipinhole projection data are acquired at multiple magnifications, with amount of multiplexing varying with magnification.

\section{DETECTORS}

The advancement of new imaging detector approaches has been driven in part by the demand for improved small-animal imaging capabilities. Despite some differences, all multipinhole collimation approaches can benefit from higher intrinsic detector resolution. As can be seen from Equation 1, improvements in detector resolution lower the magnification required for a desired image resolution, allowing for either reductions in multiplexing for a given pinhole configuration or an increase in the number of pinholes. Alternatively, the reduced area needed to accommodate the projection through a single pinhole means smaller detectors can be used. These considerations have led investigators to explore alternatives to conventional $\gamma$-cameras. Beyond the practical appeal of more compact systems, this aspect means that it is possible to explore new detector technologies that may be expensive or difficult to create in large format or number. We can provide here only a brief summary of some of these approaches. For greater detail, the interested reader is referred to the recent review article on SPECT detectors (10).

Just as in clinical SPECT, continuous scintillator crystals read out with multiple photomultiplier tubes play an important role in preclinical SPECT. Although position estimation based on Anger logic continues to be used, increased computational power has enabled practical implementation of maximumlikelihood estimation, offering improvements in performance for continuous-crystal detectors (11). Alternatively, $\gamma$-cameras based on pixelated arrays of scintillator crystals can provide spatial resolution of around $1 \mathrm{~mm}$ through the confinement of the scintillation light to an individual crystal within the array. Besides the practical difficulties of assembling an array with crystals of ever smaller dimensions, attempts to achieve better spatial resolution through the use of smaller crystals are faced with reduced detection efficiency due to the unavoidable gaps between crystals, as well as degradation in energy resolution from poor light collection efficiency. Because the position estimation also depends on generating sufficiently large signals to distinguish one crystal from another, a balance must be found between the size of the crystals and the ability to reliably identify in which crystal an interaction occurred.

Another scintillator-based approach is to use a charge-coupled device or complementary metal-oxide semiconductor to collect the scintillation light, with several different means of transmitting the scintillation light from crystal to charge-coupled device having been used, including fiber-optic tapers and lenses. Detector systems of this type have demonstrated intrinsic spatial resolution of less than $100 \mu \mathrm{m}(12-14)$. The energy resolution in such approaches tends to be relatively poor, and often the outstanding spatial resolution is achieved using thin scintillators, requiring a sacrifice in detection efficiency.

Semiconductor detectors also have received considerable attention for preclinical SPECT due to their outstanding energy resolution and ability to provide good spatial resolution via highly segmented readout electrodes. Although most of the effort for SPECT applications has focused on cadmium zinc telluride, with cadmium telluride also receiving considerable attention, our own efforts have focused on 2 other semiconductors, silicon and germanium, that offer some interesting advantages along with some challenges. Although high degrees 
of detector segmentation are possible, the chief drawback is the increase in readout channels required as detector electrodes shrink in size. The silicon detectors that we use, shown in Figure $2 \mathrm{~A}$, have a double-sided strip configuration, with the electrodes on the front and back divided into strips that are laid out orthogonally on the 2 sides. Photon interaction position is estimated in 2 dimensions by the intersection of the front and back strips on which charge is collected. This enables reporting of $N^{2}$ positions by reading out only $2 N$ strips. The silicon detectors with which we have been working are $1 \mathrm{~mm}$ thick and consist of $1,024 \times$ 1,024 strips at a pitch of $59 \mu \mathrm{m}$, effectively providing an intrinsic resolution of $59 \mu \mathrm{m}$ (Fig. 2B) (11). Two additional features make them ideal for synthetic collimator imaging. First, the double-sided strip format means that the electrical connections and associated readout electronics can be located outside the detector, enabling mounting in a transmission configuration. The second key feature is the modest detection efficiency of silicon in the 30-keV energy range. Although the approximately $30 \%$ absorption of ${ }^{125} \mathrm{I}$ photons in $1 \mathrm{~mm}$ of silicon seems less than desirable, the fact that approximately $70 \%$ proceed through the detector unaffected, taken along with the transmission configuration, means that silicon detectors can be stacked one behind the other. This stacking increases the total detection efficiency and enables multipinhole projection data to be acquired at multiple magnifications simultaneously.

More recently, we have begun investigating the potential of germanium double-sided strip detectors for small-animal SPECT. Although the excellent energy resolution has long made it an appealing material for SPECT because of the possibility of effective scatter rejection and multi-isotope imaging, segmentation of electrodes on germanium is difficult compared with other

A

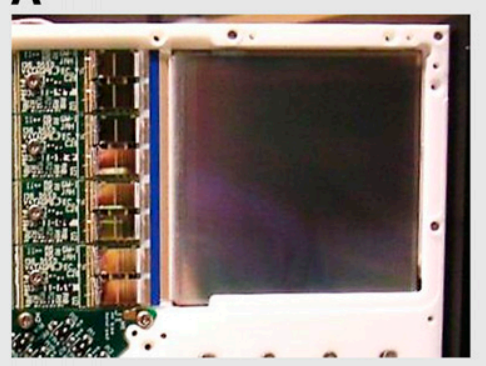

B

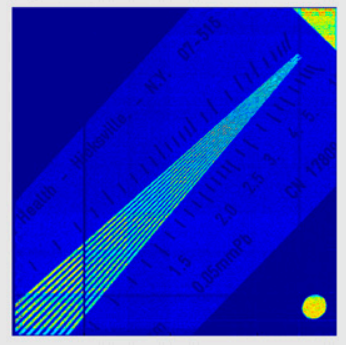

C

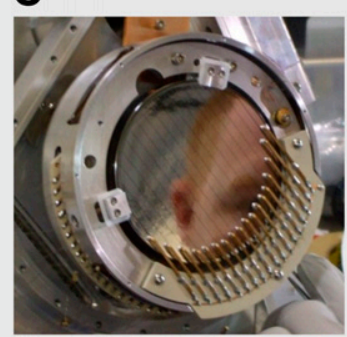

D

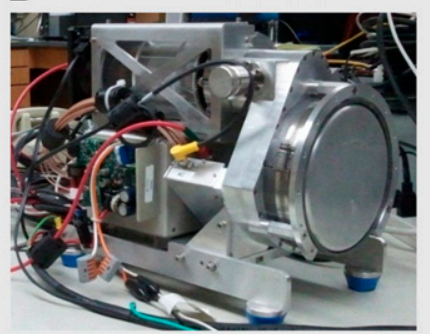

FIGURE 2. (A) Photograph of silicon double-sided strip detector in which high-density readout electronics for one side can be seen on left. (B) Shadow image of radiography line-pair phantom placed in front of silicon detector and illuminated with 125 source. (C and D) Photographs of germanium double-sided strip detector $(\mathrm{C})$ and entire detector system including readout electronics and mechanical cooling system (D). semiconductors, and the requirement of operation at liquid nitrogen temperatures has been seen as undesirable. Recent technologic advances have given rise to compact double-sided strip detector systems using mechanical cooling, rendering liquid nitrogen dewars unnecessary (Figs. 2C and 2D). The outstanding charge collection that enables such good energy resolution also facilitates the use of position-estimation techniques to achieve substrip spatial resolution along with estimation of the depth of interaction. The depth determination is based on the rise-time differences between the signals on the front and back sides, whereas the lateral positioning can be estimated from the ratio of the fast transient signals induced on the neighboring strips as charges drift toward the charge-collecting strip. Using this approach, we have been able to demonstrate intrinsic resolution of better than $1.5 \mathrm{~mm}$ in all 3 dimensions while also achieving less than $1 \%$ full-width-at-half-maximum energy resolution at 140 $\mathrm{keV}$ with a detector consisting of $16 \times 16$ strips at a pitch of 5 $\mathrm{mm}$ on a crystal that is $1 \mathrm{~cm}$ thick and $9 \mathrm{~cm}$ in diameter (15). Some sense of the potential of this detector technology for multiisotope imaging can be inferred from the pulse-height spectrum shown in Figure 3.

\section{ACQUISITION GEOMETRY}

SPECT acquisition generally involves the rotation of single or multiple camera heads in a circular orbit. Sometimes helical orbits are used to improve the angular sampling completeness at short distances (16). Stationary multipinhole SPECT is desirable because it eliminates motion-related errors, reduces geometryrelated calibration errors, and enables dynamic imaging. One difficulty in stationary SPECT is that limited angular sampling in compact systems can result in streak artifacts and geometric distortion. The FastSPECT II system of the University of Arizona successfully uses a full-ring stationary configuration (17), whereas the U-SPECT systems use stationary cameras with linear translation of the subject to improve sampling (4). Our studies have shown that with the synthetic collimator approach, high angular-sampling completeness can be achieved in a mouse-brain-sized field of view using only 2 stationary camera heads in an orthogonal geometry (9).

\section{CALIBRATION AND RECONSTRUCTION}

Efforts to provide the needed spatial resolution and sensitivity for preclinical SPECT have placed new demands on both system calibration and image reconstruction algorithms. The issues and methods to address them have been clearly described (18). Iterative statistical algorithms (19) are the standard approach for image reconstruction in preclinical SPECT. Iterative algorithms use a model of the imaging system to forward-project an object estimate, compare it with experimental projection data, and iteratively update the estimate of the object distribution. These algorithms allow for accurate accounting of pinhole geometry and photon penetration effects. Furthermore, iterative methods can include realistic models of detector resolution either by including the depth-of-interaction blurring or by including the depth-of-interaction information from detectors that have such capabilities. On the calibration front, achieving submillimeter resolution requires precise knowledge of the relative locations 


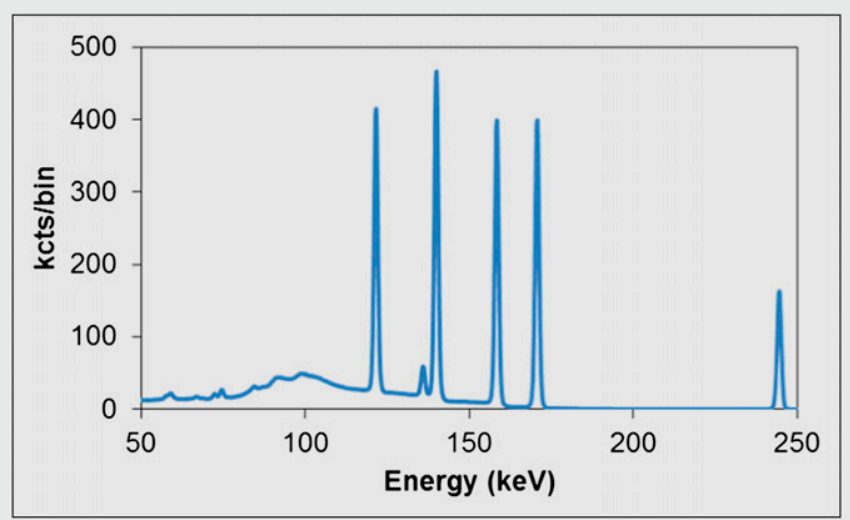

FIGURE 3. Germanium pulse-height spectrum when simultaneously exposed to ${ }^{57} \mathrm{Co}(122$ and $136 \mathrm{keV}),{ }^{99 \mathrm{mTC}}(140 \mathrm{keV}),{ }^{123} \mathrm{I}(159 \mathrm{keV})$, and ${ }^{111} \mathrm{In}$ (171 and $245 \mathrm{keV}$ ).

of the pinholes and detectors. In systems using rotating cameras, it has been found necessary to account for mechanical deviations in the camera orbits that are of negligible importance in clinical SPECT because of the substantially poorer spatial resolution (20).

The most accurate, yet tedious, way to obtain the system model is to directly measure it by stepping a point source through the field of view, thereby fully accounting for the geometry and for collimator and detector responses. Similarly, the system model can be obtained within a reasonable approximation through a Monte Carlo simulation, eliminating practical difficulties related to experimental measurements. With either method, the need for sufficient statistical quality in the system model requires lengthy acquisition or simulation time. One can also calculate the system model using analytic models for collimator sensitivity and detector response. Despite limitations, this approach is essential for design studies, where a wide range of system parameters and their impact on image quality need to be systematically investigated.

Attenuation and scatter are not as significant in small-animal SPECT as in clinical SPECT and therefore are often neglected in image reconstruction. It has been shown that performing attenuation correction without scatter correction can lead to a significant overestimation of activity concentration but that high quantitative accuracy can be achieved by correcting for both effects $(21,22)$. Partial-volume errors also can lead to significant underestimation of activity concentration in many small-animal SPECT scenarios, providing impetus to improve resolution through smaller pinholes, higher magnification, or better detector resolution. However, if gains in resolution come at too large a cost in sensitivity, the improvement in quantitative accuracy can be accompanied by a decrease in precision due to greater noise.

\section{PRESENT STATUS}

The demand for preclinical SPECT capabilities, as well as the demands placed on the instrumentation to create useful preclinical SPECT images, has led to considerable innovation. Quantitative images can now be obtained with submillimeter spatial resolution at a practical sensitivity. Furthermore, some of the technologies first demonstrated in preclinical SPECT are beginning to find use in clinical imaging. Cadmium zinc telluride detectors can now be found in dedicated cardiac SPECT systems
$(23,24)$, and both cadmium zinc telluride detectors and pixelated scintillators are used for breast imaging $(25,26)$. Multipinhole approaches are under investigation for brain imaging (27) and have been used in a static, limited-angle configuration for cardiac imaging (24).

\section{ACKNOWLEDGMENTS}

This work was supported in part by NIH grants R33EB00776, R01EB013677, and K99EB009106 and by Department of Energy grant DE-SC0002437. We gratefully acknowledge the important contributions of Desmond L. Campbell, Heather L. Durko, Lindsay C. Johnson, Benjamin S. McDonald, and Oleg Ovchinnikov to the silicon and germanium detector work. No other potential conflict of interest relevant to this article was reported.

\section{REFERENCES}

1. Shokouhi S, Metzler SD, Wilson DW, Peterson TE. Multi-pinhole collimator design for small-object imaging with SiliSPECT: a high-resolution SPECT. Phys Med Biol. 2009;54: 207-225.

2. Vunckx K, Suetens P, Nuyts J. Effect of overlapping projections on reconstruction image quality in multipinhole SPECT. IEEE Trans Med Imaging. 2008;27:972-983.

3. Mok GS, Tsui BM, Beekman FJ. The effects of object activity distribution on multiplexing multi-pinhole SPECT. Phys Med Biol. 2011;56:2635-2650.

4. Vastenhouw B, Beekman F. Submillimeter total-body murine imaging with U-SPECT-I. J Nucl Med. 2007;48:487-493.

5. Schramm NU, Ebel G, Engeland U, Schurrat T, Behe M, Behr TM. High-resolution SPECT using multipinhole collimation. IEEE Trans Nucl Sci. 2003;50:315-320.

6. Rentmeester MC, van der Have F, Beekman FJ. Optimizing multi-pinhole SPECT geometries using an analytical model. Phys Med Biol. 2007;52:2567-2581.

7. Cao Z, Bal G, Accorsi R, Acton PD. Optimal number of pinholes in multi-pinhole SPECT for mouse brain imaging: a simulation study. Phys Med Biol. 2005;50:4609-4624.

8. Wilson DW, Barrett HH, Clarkson EW. Reconstruction of two- and three-dimensional images from synthetic-collimator data. IEEE Trans Med Imaging. 2000;19:412-422.

9. Shokouhi S, Wilson DW, Metzler SD, Peterson TE. Evaluation of image reconstruction for mouse brain imaging with synthetic collimation from highly multiplexed SiliSPECT projections. Phys Med Biol. 2010;55:5151-5168.

10. Peterson TE, Furenlid LR. SPECT detectors: the Anger camera and beyond. Phys Med Biol. 2011;56:R145-R182.

11. Barrett HH, Hunter WCJ, Miller BW, Moore SK, Chen Y, Furenlid LR. Maximum-likelihood methods for processing signals from gamma-ray detectors. IEEE Trans Nucl Sci. 2009;56: 725-735.

12. Meng LJ, Fu G. Investigation of the intrinsic spatial resolution of an intensified EMCCD scintillation camera. IEEE Trans Nucl Sci. 2008;55:2508-2517.

13. Miller BW, Barrett HH, Furenlid LR, Barber HB, Hunter RJ. Recent advances in BazookaSPECT: real-time data processing and the development of a gamma-ray microscope. Nucl Instrum Methods Phys Res A. 2008;591:272-275.

14. Korevaar MA, Heemskerk JW, Goorden MC, Beekman FJ. Multi-scale algorithm for improved scintillation detection in a CCD-based gamma camera. Phys Med Biol. 2009:54:831-842.

15. Johnson LC, Campbell DL, Hull EL, Peterson TE. Characterization of a high-purity germanium detector for small-animal SPECT. Phys Med Biol. 2011;56:5877-5888.

16. Metzler SD, Greer KL, Jaszczak RJ. Helical pinhole SPECT for small-animal imaging: a method for addressing sampling completeness. IEEE Trans Nucl Sci. 2003;50:1575-1583.

17. Furenlid LR, Wilson DW, Chen YC, et al. FastSPECT II: a second-generation high-resolution dynamic SPECT imager. IEEE Trans Nucl Sci. 2004;51:631-635.

18. Nuyts J, Vunckx K, Defrise M, Vanhove C. Small animal imaging with multi-pinhole SPECT. Methods. 2009;48:83-91

19. Qi J, Leahy RM. Iterative reconstruction techniques in emission computed tomography. Phys Med Biol. 2006;51:R541-R578.

20. Defrise M, Vanhove C, Nuyts J. Perturbative refinement of the geometric calibration in pinhole SPECT. IEEE Trans Med Imaging. 2008;27:204-214.

21. Chen CL, Wang Y, Lee JJ, Tsui BM. Toward quantitative small animal pinhole SPECT: assessment of quantitation accuracy prior to image compensations. Mol Imaging Biol. 2009;11:195-203.

22. Vanhove $\mathrm{C}$, Defrise $\mathrm{M}$, Bossuyt $\mathrm{A}$, Lahoutte T. Improved quantification in single-pinhole and multiplepinhole SPECT using micro-CT information. Eur J Nucl Med Mol Imaging. 2009;36:1049-1063.

23. Gambhir SS, Berman DS, Ziffer J, et al. A novel high-sensitivity rapid-acquisition singlephoton cardiac imaging camera. J Nucl Med. 2009;50:635-643.

24. Bocher M, Blevis I, Tsukerman L, Shrem Y, Kovalski G, Volokh L. A fast cardiac gamma camera with dynamic SPECT capabilities: design, system validation and future potential. Eur $J$ Nucl Med Mol Imaging. 2010;37:1887-1902.

25. Hruska CB, Phillips SW, Whaley DH, Rhodes DJ, O'Connor MK. Molecular breast imaging: use of a dual-head dedicated gamma camera to detect small breast tumors. AJR. 2008;191:1805-1815.

26. Brem RF, Michener KH, Zawistowski G. Approaches to improving breast cancer diagnosis using a high resolution, breast specific gamma camera. Phys Med. 2006;21(suppl 1):17-19.

27. Goorden MC, Rentmeester MC, Beekman FJ. Theoretical analysis of full-ring multi-pinhole brain SPECT. Phys Med Biol. 2009;54:6593-6610. 